\title{
Efectividad del tratamiento médico con misoprostol según dosis administrada en aborto retenido menor de 12 semanas
}

\author{
Joaquín Errázuriz V. ${ }^{1}$, Milena Stambuk B. ${ }^{1}$, Felipe Reyes $Z{ }^{a}{ }^{\text {, Francisco Sumar U. }}{ }^{a}$, \\ Carlos Rondini F-D. ${ }^{1}$, Fernando Troncoso R. ${ }^{1}$ \\ 1 Departamento de Ginecología, Unidad de Gestión Clínica de la Mujer y el Recién Nacido. Hospital Padre Hurtado, \\ Facultad de Medicina Clínica Alemana-Universidad del Desarrollo, Santiago, Chile.
}

a Alumna/o, Escuela de Medicina, Universidad del Desarrollo.

\section{RESUMEN}

Antecedentes: El manejo terapéutico del aborto retenido consiste en evacuar la cavidad uterina espontáneamente o utilizando misoprostol previo al legrado quirúrgico. Objetivo: Evaluar la necesidad de dilatación mecánica post maduración cervical con misoprostol y la tasa de perforación uterina post legrado, utilizando diferentes dosis de misoprostol en pacientes con diagnóstico de aborto retenido menor a 12 semanas. Métodos: Se registraron datos demográficos y ginecológicos de una cohorte retrospectiva de pacientes con diagnóstico de aborto retenido menor a 12 semanas, entre enero de 2008 y diciembre de 2010. Se establecieron 3 grupos de trabajo según la dosis de misoprostol administrada vía vaginal, siendo de $100(n=131), 200(n=231)$ y $400 \mu g(n=230)$, y se observaron las complicaciones asociadas al procedimiento. Resultados: La necesidad de dilatación mecánica fue significativamente mayor en el grupo que recibió $100 \mu \mathrm{g}$ de misoprostol al compararlo con el de $200 \mu \mathrm{g}$ y $400 \mu \mathrm{g}(\mathrm{p}<0,01)$. No hubo diferencias estadísticamente significativas entre las que recibieron 200 versus $400 \mu \mathrm{g}$ de misoprostol. No hubo diferencias significativas respecto a perforación uterina. Conclusión: En el aborto retenido menor a 12 semanas, la necesidad de dilatación mecánica post maduración cervical, es menor si se utiliza 200 o 400 $\mu \mathrm{g}$ de misoprostol, sin diferencias en la tasa de perforación uterina.

\section{PALABRAS CLAVE: Aborto retenido, misoprostol, dilatación cervical}

\section{SUMMARY}

Background: The therapeutic management of missed abortion consists on evacuating the uterine cavity, spontaneously or by administration of misoprostol previous to curettage. Objectives: Evaluate the need of mechanical dilatation after cervical maturation with misoprostol and the rate of uterine perforation before curettage, using different doses of misoprostol in patients with diagnosis of missed abortion before 12 weeks. Methods: Demographic and gynecologic data were registered of a retrospective cohort of patients with the diagnosis of missed abortion before 12 weeks, between January 2008 and December 2010. Three groups were established according to the dose of misoprostol: $100(n=131), 200(n=231)$ and $400 \mu g(n=230)$. Complications associated to the procedure were observed. Results: The need of mechanical dilatation was significant higher for the group with $100 \mu \mathrm{g}$ of misoprostol in comparison with 200 and $400 \mu \mathrm{g}(\mathrm{p}<0.001)$. 
There was no statistical significance among who received 200 versus $400 \mu \mathrm{g}$ of misoprostol. No statistical significance was found for uterine perforation. Conclusion: In the missed abortion before 12 week, the need of mechanical dilatation is lower with 200 or $400 \mu \mathrm{g}$ of misoprostol, without difference in uterine perforation rate.

\section{KEYS WORDS: Missed abortion, misoprostol, cervical dilatation}

\section{INTRODUCCIÓN}

El aborto retenido es una condición clínica que ocurre aproximadamente en un $20 \%$ de los embarazos de primer trimestre (1). El manejo puede ser expectante, médico o quirúrgico. El primero consiste en permitir que el útero expulse espontáneamente el contenido, sin intervención. Estudios recientes concluyen que el porcentaje de éxito con manejo expectante, es más bajo que el médico o quirúrgico (2), y se asoció a un mayor riesgo de aborto incompleto, necesidad de evacuación quirúrgica y hemorragia (3).

El manejo médico se realiza utilizando misoprostol, un análogo de prostaglandina $\mathrm{E} 1$, que posee propiedades útero constrictoras y abortivas (4). Este medicamento ha sido ampliamente documentado, como tratamiento de aborto retenido menor o igual a 12 semanas (5). Diversos estudios comparan el tratamiento médico con la intervención quirúrgica para el manejo del aborto espontáneo $(6,7)$. La media de éxito del misoprostol y cirugía fue $92 \%$ y $98 \%$ respectivamente. Weeks y cols (8), obtuvieron una tasa de éxito del $96,3 \%$ con misoprostol, mientras que con el tratamiento quirúrgico fue de $91,5 \%$. Si bien los procedimientos quirúrgicos de evacuación presentan una elevada tasa de éxito, no están exentos de complicaciones como infección, laceración cervical, hemorragia y perforación uterina, con tasas de mortalidad de 0,05\% (9). En general, el misoprostol puede ser usado para la maduración cervical previa a abortos quirúrgicos, en mujeres con cesárea anterior en el primer o segundo trimestre, porque la ruptura uterina rara vez ocurre en este marco (10).

El objetivo de este estudio es analizar la necesidad de dilatación post maduración cervical con misoprostol vaginal a diferentes dosis y la tasa de perforación uterina post legrado, en pacientes con diagnóstico de aborto retenido menor a 12 semanas.

\section{PACIENTES Y MÉTODOS}

Estudio retrospectivo, que utilizó la información almacenada en el sistema informático de la Unidad de Ginecología del Hospital Padre Hurtado, Santiago, Chile. Se incluyeron a todas las pacientes con diagnóstico de aborto retenido menor de 12 semanas atendidas en la institución, en el período entre enero de 2008 a diciembre de 2010. Se registraron características demográficas de la población en estudio y datos ginecológicos de paridad, número de abortos previos, dosis de misoprostol administrada, fracaso de manejo médico y perforación uterina asociado a manejo quirúrgico. No se realizó análisis de diferencias en pacientes con antecedentes de cicatriz previa anterior.

Se establecieron 3 grupos de trabajo según dosis de misoprostol administrada vía vaginal: 100, 200 o $400 \mu \mathrm{g}$. Se definió como fracaso médico, pacientes que requirieron dilatación cervical mecánica al menos 6 horas post administración de misoprostol y a todas se les realizó legrado posteriormente. El análisis estadístico se hizo utilizando la prueba de Chi-cuadrado. Se consideró diferencia significativa un valor $p<0,05$

\section{RESULTADOS}

Durante el período estudiado, hubo un total de 592 pacientes con diagnóstico de aborto retenido menor a 12 semanas. El promedio de edad fue de 23,7 años (rango: 16-46 años), 474 (80\%) correspondieron a mujeres menores de 40 años y 118 $(20 \%)$ a mayores de 40 años.

Se administró vía vaginal, $100 \mu \mathrm{g}$ de misoprostol a 131 pacientes, $200 \mu \mathrm{g}$ a 231 pacientes y $400 \mu \mathrm{g}$ a 230 pacientes (Tabla I). La tasa total de fracaso terapéutico fue de $5,7 \%$ (34/592). El análisis por grupo fue de $16 \%$ (21/131), 2,2\% (5/231) y $3,5 \%(8 / 230)$, respectivamente, según dosis administrada. El análisis univariado para evaluar la necesidad de dilatación mecánica post maduración cervical, encontró diferencias significativas entre las pacientes que recibieron $100 \mu \mathrm{g}$ de misoprostol y las que recibieron 200 y $400 \mu \mathrm{g}$, respectivamente $(p<0,01)$. No hubo diferencias significativas entre las pacientes que recibieron 200 y $400 \mu \mathrm{g}$ de misoprostol $(p=0,2)$ (Figura 1). 
Hubo 4 pacientes que se complicaron con perforación uterina, con una tasa de 1,5\% (2/131), $0 \%(0 / 231)$ y de $0,8 \%(2 / 230)$ para pacientes que recibieron 100, 200 y $400 \mu \mathrm{g}$ de misoprostol, respectivamente, sin diferencias significativas entre los grupos $(p=0,4)$. No hubo mortalidad en nuestra serie.

Tabla I ANÁLISIS DE GRUPOS QUE REQUIRIERON
DILATACIÓN MECÁNICA CON DIFERENTES
DOSIS DE MISOPROSTOL

\begin{tabular}{|c|c|c|c|c|c|}
\hline \multirow{3}{*}{$\begin{array}{c}\text { Misoprostol } \\
(\mu \mathrm{g})\end{array}$} & \multicolumn{4}{|c|}{ Dilatación } & \multirow{3}{*}{$\begin{array}{c}\text { Total } \\
\mathrm{n}\end{array}$} \\
\hline & \multicolumn{2}{|c|}{ No } & \multicolumn{2}{|c|}{$\mathrm{Si}$} & \\
\hline & $n$ & $\%$ & $\mathrm{n}$ & $\%$ & \\
\hline 100 & 110 & 84 & 21 & 16 & 131 \\
\hline 200 & 226 & 97,8 & 5 & 2,2 & 231 \\
\hline 400 & 222 & 96,5 & 8 & 3,5 & 230 \\
\hline Total & 558 & 94,3 & 34 & 5,7 & 592 \\
\hline
\end{tabular}

\section{DISCUSIÓN}

La disponibilidad de tratamientos médicos para inducir pacientes con aborto retenido ha creado nuevas opciones de manejo. Misoprostol, es el agente más utilizado como tal. La seguridad y eficacia han sido establecidas por múltiples ensayos aleatorizados y controlados. Las ventajas sobre otros fármacos son su bajo costo, fácil disponibilidad y baja incidencia de complicaciones (11). Los efectos secundarios descritos más frecuentes son: fiebre, escalofríos, dolor abdominal, sensación de náuseas y mareos, que ocurren con mayor frecuencia cuando es administrado vía oral (12). Trinder y cols (13), en un ensayo controlado y randomizado sobre el manejo del aborto espontáneo con tratamiento médico, quirúrgico o expectante, no encontró diferencias significativas en la incidencia de infección o eventos adversos serios entre los tres métodos.

La eficacia del tratamiento médico con misoprostol depende de la dosis y vía de administración, pero

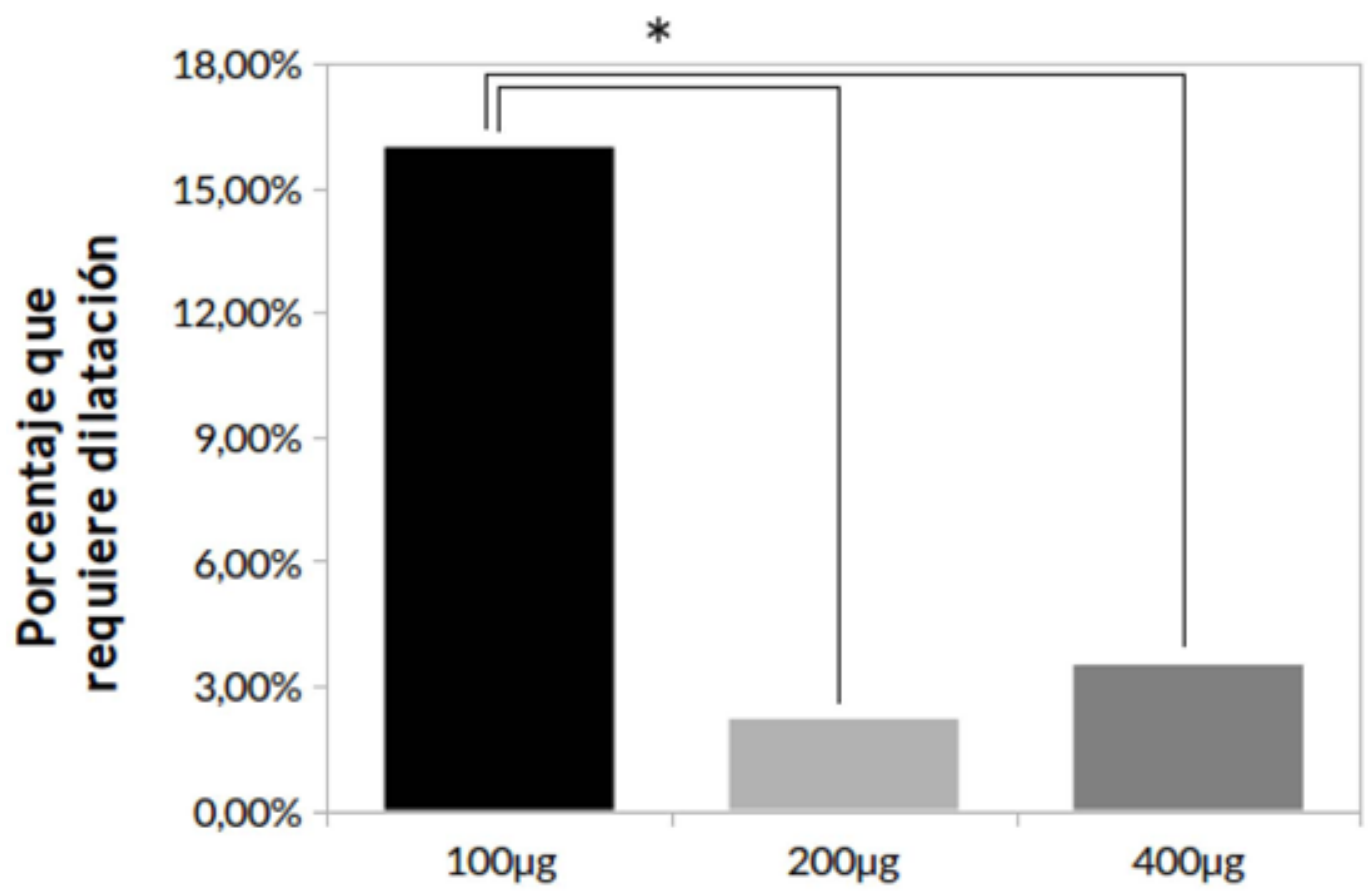

\section{Dosis Misoprostol}

${ }^{*} \mathrm{p}<0,05$ (Chi cuadrado)

Figura 1. Eficiencia de misoprostol a diferentes dosis. 
no hay consenso sobre la elección óptima. Estudios norteamericanos comparan el manejo médico con dosis oral única de $400 \mu \mathrm{g}$ de misoprostol contra igual dosis oral cada 4 horas, obteniendo tasas de éxito del $13 \%$ versus $50-70 \%$ respectivamente (14). La administración oral parece ser tan efectiva como la administración vaginal, pero se asocia con más efectos secundarios, probablemente relacionadas con diferencias en la farmacocinética (15).

Estudios prospectivos, comparan misoprostol solo y en combinación con mifepristona, obteniendo tasas similares de éxito en aborto retenido, sin embargo la adición de un antagonista de la progesterona es cuestionable y de alto costo (16). Neilson y cols (17), obtuvieron una tasa de expulsión utilizando dosis de 600 a $800 \mu$ g por vía vaginal de 70 a 90\%. Estudios europeos alcanzaron cifras cercanas al $46 \%$ y $66 \%$ de abortos completos con dosis de $200 \mu \mathrm{g}$ y 400 $\mu \mathrm{g}$ de misoprostol, respectivamente (18). Rabey cols (19), en la interrupción del embarazo entre las 9 y 12 semanas, con 2 dosis de 200 o $400 \mu \mathrm{g}$ de misoprostol, obtuvieron aborto parcial/completo de 9 y $11 \%$ respectivamente.

La perforación uterina es una complicación severa, si bien su incidencia es baja, cuando ocurre, el diagnóstico debe ser precoz y el manejo oportuno, con uso de retractores uterinos, terapia médica con antibióticos y en ciertas oportunidades, resolución quirúrgica (20). El porcentaje de perforación uterina fue menor al $1 \%$, siendo similar a lo publicado en la literatura (21).

Varios estudios han demostrado que la dosis óptima en términos de balance efectividad-efectos secundarios es $400 \mu \mathrm{g}$ (22). Se ha evaluado la vía de administración oral, vaginal y sublingual. Son regímenes efectivos: $400 \mu \mathrm{g}$ de misoprostol vaginal administrados 3-4 horas antes del procedimiento quirúrgico evacuador, $400 \mu \mathrm{g}$ oral $8-12$ horas antes, ó $400 \mu \mathrm{g}$ sublingual 2-4 horas antes (23). En nuestra serie hubo mayores tasas de fracaso del manejo médico en el grupo de pacientes que recibió dosis de $100 \mu \mathrm{g}$ misoprostol, y la tasa de éxito con dosis única de misoprostol de $200 \mathrm{gg}$ y $400 \mathrm{gg}$, a las 6 horas de su aplicación vaginal fue de $97,8 \%$ y $96,5 \%$ respectivamente. No se encontró mayor riesgo de perforación uterina en ninguno de los grupos. Si bien no se evaluaron efectos secundarios atribuibles a la droga, según dosis administrada, no se reportaron complicaciones mayores ni muertes maternas.

A pesar de esta evidencia, actualmente en Chile no existen protocolos que indiquen dosis seguras y eficaces para el manejo de aborto retenido, se requieren estudios prospectivos que comparen las dosis de misoprostol con mayor número de pacientes que permita obtener resultados estadísticos que determinen la efectividad, además de evaluar la incidencia de otras posibles complicaciones propias del medicamento y variables que determinen las tasas de éxito.

\section{CONCLUSIÓN}

La necesidad de dilatación mecánica post maduración cervical, en pacientes con diagnóstico de aborto retenido menor a 12 semanas, es menor si se utiliza 200 o $400 \mu \mathrm{g}$ de misoprostol, sin aumento de la tasa de perforación uterina por legrado uterino.

\section{REFERENCIAS}

1. Jurkovic D, Overton C, Bender-Atik R. Diagnosis and management of first trimester miscarriage. BMJ 2013;346:f3676.

2. Sotiriadis A, Makrydimas G, Papatheodorou $S$, loannidis JP. Expectant, medical or surgical management of first-trimester miscarriage: A metaanalysis. Obstet Gynecol 2005;105:1104-13.

3. Nanda K, Peloggia A, Grimes D, Lopez L, Nanda G. Expectant care versus surgical treatment for miscarriage. Cochrane Database Syst Rev 2006;CD003518.

4. Norman JE, Thong KJ. Uterine contractility and induction of abortion in early pregnancy by misoprostol and mifepristone. Lancet 1997;338:1233-6.

5. Blum J, Winikoff B, Gemzell-Danielsson K, Ho PC, Schiavon R, Weeks A. Treatment of incomplete abortion and miscarriage with misoprostol. Int $\mathrm{J}$ Gynaecol Obstet 2007;99:S186-S189.

6. Demetroulis C, Saridogan E, Kunde D, Naftalin AA. A prospective randomized control trial comparing medical and surgical treatment for early pregnancy failure. Human Reprod 2001;16:365-9.

7. Sahin HG, Sahin HA, Kocer M. Randomized outpatient clinical trial of medical evacuation and surgical curettage in incomplete miscarriage. Eur J Contracep Reprod Health Care 2001;6:141-4.

8. Weeks A, Alia G, Blum J, Winikoff B, Ekwaru P, Durocher $\mathrm{J}$, et al. A randomized trial of misoprostol compared with manual vacuum aspiration for incomplete abortion. Obstet Gynecol 2005;106:540-7.

9. Harris LH, Dalton VK, Johnson TR. Surgical management of early pregnancy failure: history, politics, and safe, cost-effective care. Am J Obstet Gynecol 2007;196:445.e1-445.e5.

10. Fox MC, Hayes JL; Society of Family Planning. Cervical preparation for second- trimester surgical abortion prior to 20 weeks of gestation. Contraception 2007;76:486-95.

11. Graziosi GC, van der Steeg JW, ReuwerPH,Drogtrop AP, Bruinse HW, Mol BW. Economic evaluation of misoprostol in the treatment of early pregnancy failure compared to curettage after an expectant management. Hum Reprod 2005;20:1067-71. 
12. Weeks A, Faúndes A. Misoprostol in obstetrics and gynecology. Int J Gynaecol Obstet 2007;99:S156-S159.

13. Trinder J, Brocklehurst $P$, Porter R, Read M, Vyas $\mathrm{S}$, Smith L. Management of miscarriage: expectant, medical, or surgical? Results of randomized controlled trial (miscarriage treatment (MIST) trial). BMJ 2006;332:1235-40.

14. Chung T, Leung P, Cheung LP, Haines $C$, Chang AM. A medical approach to management of spontaneous abortion using misoprostol. Extending misoprostol treatment to a maximum of 48 hours can further improve evacuation of retained products of conception in spontaneous abortion. Acta Obstet Gynecol Scand 1997;76:248-51.

15. Zieman M, Fong SK, BenowitzNL,Banskter D, Darney PD. Absorption kinetics of misoprostol with oral or vaginal administration. Obstet Gynecol 1997;90:88-92.

16. Stockheim D, Machtinger R, Wiser A, Dulitzky M, Soriano D, Goldenberg M, et al. A randomized prospective study of misoprostol or mifepristone followed by misoprostol when needed for the treatment of women with early pregnancy failure. Fertil Steril 2006;86:956-60.

17. Neilson JP, Hickey M, Vazquez J. Medical treatment for early fetal death (less than 24 weeks). Cochrane Database Syst Rev 2006;CD002253.
18. Koopersmith TB, Mishell DR. The use of misoprostol for termination for early pregnancy. Contraception 1996;53:237-42.

19. Rabe T, Basse H, Thuro H, Kiesel L, Runnebaum B. Effect of PGE1 Methyl analogue misoprostol on the pregnant uterus in the first trimester. Geburtshilfe Frauenheilkd 1987;47:324-31.

20. Forna F, Gülmezoglu AM. Surgical procedures to evacuate incomplete abortion. Cochrane Database Syst Rev 2001;CD001993.

21. Meirik O, My Huong NT, Piaggio G, Bergel G, von Hertzen $\mathrm{H}$; WHO Research Group on postovulatory methods of Fertility Regulation. Complications of firsttrimester abortion by vacuum aspiration after cervical preparation with and without misoprostol: a multicentre randomized trial. Lancet 2012;379:1817-24.

22. Singh K, Fong YF. Preparation of the cervix for surgical termination of pregnancy in the first trimester. Hum Reprod Update 2000;6:442-8.

23. Allen RH, Goldberg AB. Board of Society of Family Planning. Cervical dilation before first-trimester surgical abortion ( $<14$ weeks gestation). SFP Guideline 20071. Contraception 2007;76:139-56. 